\title{
Searching for the onset of deconfinement at FAIR: the CBM experiment
}

\author{
Volker Friese ${ }^{1}$ \\ Gesellschaft für Schwerionenforschung $\mathrm{mbH}$ \\ Planckstraße 1, 64291 Darmstadt, Germany \\ E-mail:v.friese@gsi.de
}

\section{for the CBM Collaboration}

\begin{abstract}
High-energy heavy-ion collisions provide a tool to study strongly interacting matter under extreme conditions, i. e. densities and temperatures. The goal of this experimental programme is to create and study a state of matter where quarks and gluons are no longer confined into hadrons. Several experimental findings suggest that these deconfinement sets in at beam energies of about $30 \mathrm{GeV}$ per nucleon. The CBM experiment at the future facility FAIR in Darmstadt, Germany, will investigate this energy domain $(10-45 \mathrm{AGeV})$ with respect to the first-order phase transition from hadronic to partonic matter, the properties of extremely dense nuclear matter, and the critical point of QCD. Special emphasis will be put on very rare probes like charmed hadrons, multi-strange hyperons, and di-lepton spectroscopy. We will outline the planned experiment, the status of preparations, and the expected physics performances of the CBM experiment.
\end{abstract}

8th Conference Quark Confinement and the Hadron Spectrum

Mainz, Germany

1-6 September, 2008

\footnotetext{
$1 \quad$ Speaker
} 


\section{Introduction}

It is believed that above a certain critical energy density, strongly interacting matter exists in a deconfined state of quarks and gluons. Such a state can be reached by heating and/or compressing nuclear matter. In accelerator laboratories, this is done by colliding heavy nuclei, thus creating a dense and hot fireball, albeit only in a very limited space-time domain.

Results from lattice QCD [1] indicate that the transition from confined to deconfined matter at vanishing net baryon density, as encountered in nuclear collisions at RHIC or LHC energies, is a smooth crossover, while in the region of high densities, accessible with heavy-ion reactions at lower beam energies, a first-order phase transition is expected. The experimental confirmation of this first-order transition would be a breakthrough in our understanding of the properties of strongly interacting matter.

The most promising way to search for the deconfinement phase transition is to look for a non-monotonic behaviour of observables as function of collision energy and system size. The CBM experiment at the future research centre FAIR in Darmstadt is being designed to execute this search with a large range of observables, including very rare probes like open charm. It will investigate heavy-ion collision in the beam energy range $10 A-45 A \mathrm{GeV}$ from 2016 on.

\section{The CBM experiment at FAIR}

The FAIR facility at GSI [8] will offer unique possibilities for the investigation of the QCD phase diagram at extreme net-baryon densities, delivering fully stripped external heavyion beams up to uranium with intensities of up to $2 \cdot 10^{9}$ per second at beam energies from 8 to $35 \mathrm{AGeV}$. Lighter ions $(\mathrm{Z} / \mathrm{A}=0.5)$ can be accelerated up to $45 \mathrm{AGeV}$, while proton beams will be available up to $90 \mathrm{GeV}$. The unprecedented beam intensities will allow studying extremely rare probes with high precision but also constitute a high challenge for detectors and electronics.

The CBM (Compressed Baryonic Matter) experiment [9] will be a next-generation fixedtarget detector to be operated at the FAIR heavy-ion synchrotron SIS-300. It is designed to measure hadronic, leptonic and photonic probes in a large acceptance and at the extreme interaction rates offered by the accelerator. $\mathrm{CBM}$ aims at a systematic investigation of $\mathrm{A}+\mathrm{A}$, $p+A$ and $p+p$ collisions, in terms of collision energy $\left({\sqrt{\mathrm{S}_{\mathrm{NN}}}}=4.5-9.3 \mathrm{GeV}\right.$ for heavy nuclei) and system size, with high precision and statistics. In contrast to the low-energy programmes at the RHIC and the SPS, which due to low collision rates will focus on bulk particle production, CBM will put special emphasis on the measurement of extremely rare probes which have not been accessible by previous heavy-ion experiments at the AGS and the SPS. 

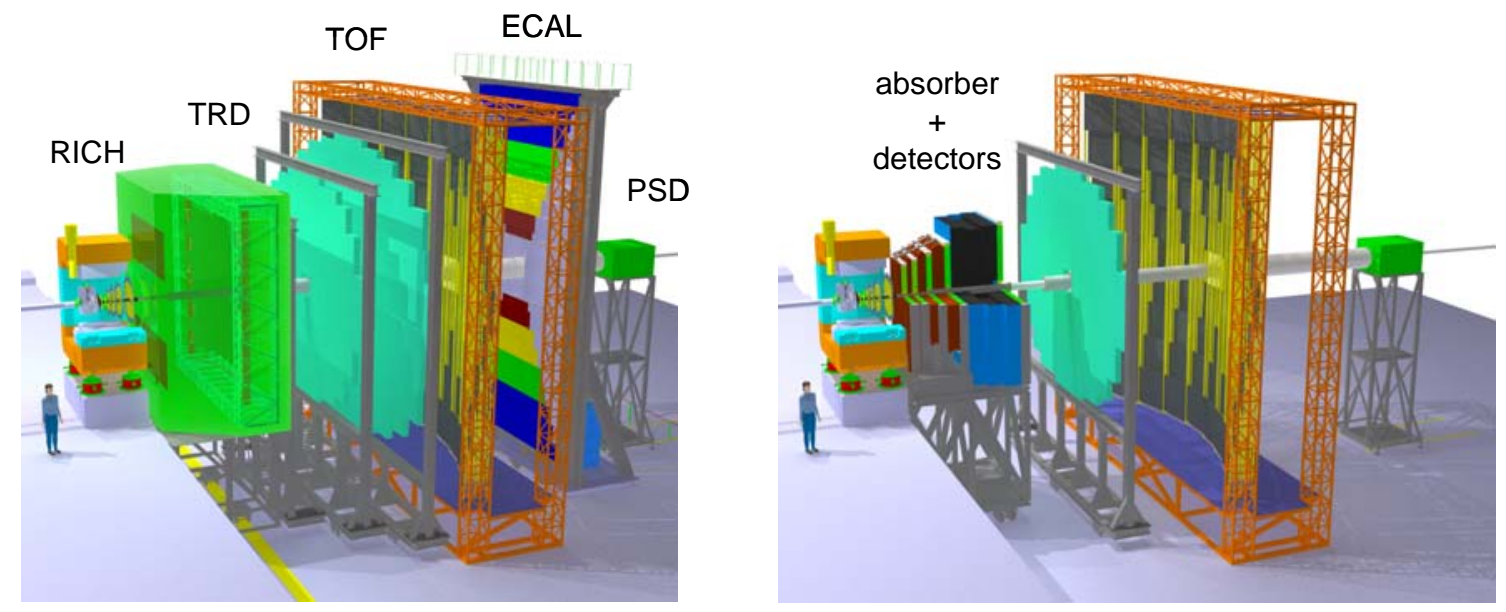

Figure 1. Schematic layout of the CBM experiment. Left: configuration for electron measurements; right: configuration for muon measurements.

The observables to be covered by CBM include multiplicities, phase space distributions and flow of strange and multi-strange hadrons $(\mathrm{K}, \varphi, \Lambda, \Xi, \Omega)$ and charmed hadrons $\left(\mathrm{D}, \mathrm{D}_{\mathrm{s}}, \Lambda_{\mathrm{c}}\right)$. The in-medium properties of short-lived vector mesons $(\varphi, \omega, \varphi)$ will be investigated via their di-leptonic decay. The measurement of lepton pairs will also give access to charmonium states which, together with the open charm measurements, will allow a comprehensive study of charm production near threshold. Signatures of the critical point will be looked for in event-by-event fluctuations of quantities like particle yield ratios, charged multiplicity or average $\mathrm{p}_{\mathrm{t}}$.

To cope with these requirements, the CBM detector must provide hadron and lepton identification in a large acceptance as well as excellent vertexing for the open charm measurements. In addition, the extreme interaction rates needed for the rarest probes call for fast and radiation-hard detectors and read-out electronics, as well as for efficient online data processing and event selection. Currently, two configurations are being evaluated, corresponding to the measurements of electrons and muons, respectively (figure 1). The electron setup comprises a silicon tracking system (STS) inside a dipole magnet, combined with a micro-vertex detector (MVD). Electrons are identified in a ring imaging Cherenkov detector (RICH) and an array of transition radiation detectors (TRD). The setup is completed by a timeof-flight system for hadron identification and an electro-magnetic calorimeter for the detection of neutral particles. For muon measurements, the RICH detector will be replaced by an active absorber system (MUCH). 

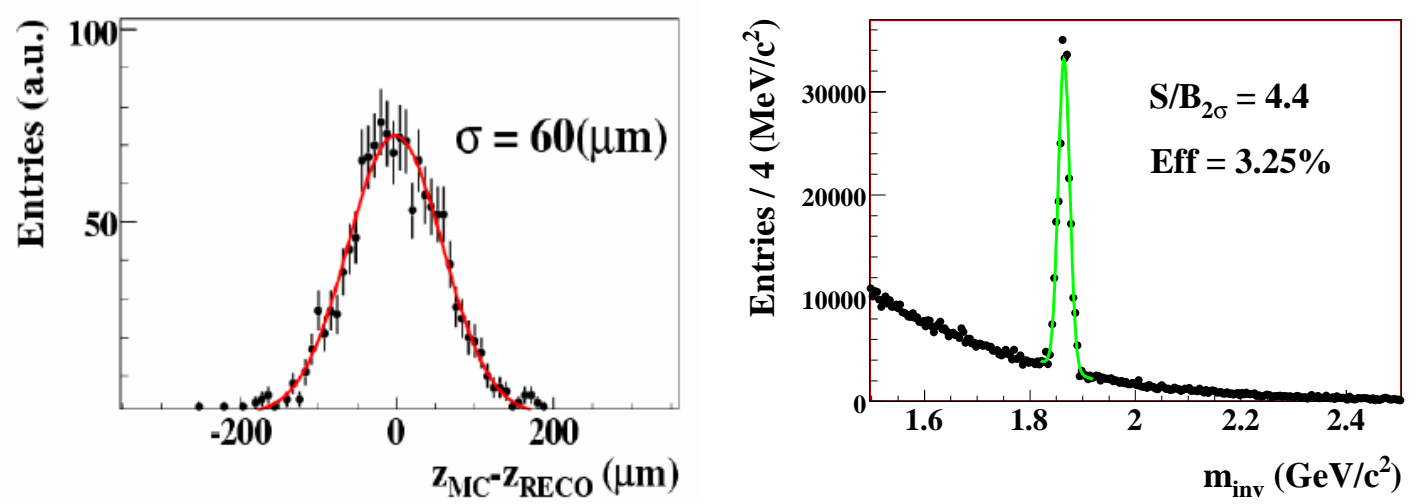

Figure 2. (Left) Distance between reconstructed and true vertex of $D^{0} \rightarrow \pi^{+} K^{-}$embedded into simulated central $\mathrm{Au}+\mathrm{Au}$ events at $25 \mathrm{AGeV}$. (Right) Reconstructed invariant-mass spectrum for the decay $D^{0} \rightarrow \pi^{+} K^{-}$

\section{Observables and physics performance}

\subsection{Open charm}

The measurement of charmed hadrons near the threshold provides a most promising tool to discriminate hadronic from partonic production scenarios, the former requiring the creation of pairs of charmed hadrons (e.g. $\mathrm{D}^{+} \mathrm{D}^{-}$or $\mathrm{D}^{0} \Lambda$ ), the latter the recombination of c quarks created in the first hard collisions of the incoming hadrons. Of particular interest are yield ratios of charmed hadrons since these are independent from the assumption of the c-cbar production cross section. However, the detection of charmed hadrons via their weak decay into kaons and pions in a background of several hundreds of directly produced charged hadrons requires an excellent detection of the displaced decay vertex with a resolution of below $100 \mu \mathrm{m}$. This task will be performed by the Micro-Vertex Detector composed of two or three ultra-thin detector layers based on monolothic active pixel sensors (MAPS) operated in vacuum close to the target. These sensor type provides a low enough material budget and sufficient coordinate resolution for high-precision vertex detection, as demonstrated in figure 2 .

\subsection{Charmonium}

Charmonium will be measured via its decay in either electron or muon pairs. Electron identification is achieved by the Ring Imaging Cherenkov (RICH) detector and several layers of Transition Radiation Detectors (TRD). The combined performance of RICH and TRD allows a pion suppression of about $10^{4}$ at electron efficiencies of about $70-80 \%$ for momenta above 1 $\mathrm{GeV}$. At lower momenta, the additional use of TOF information still improves on the rejection of pions. The remaining background consists mainly of electrons from Dalitz decays and photon conversion in the detector material. 

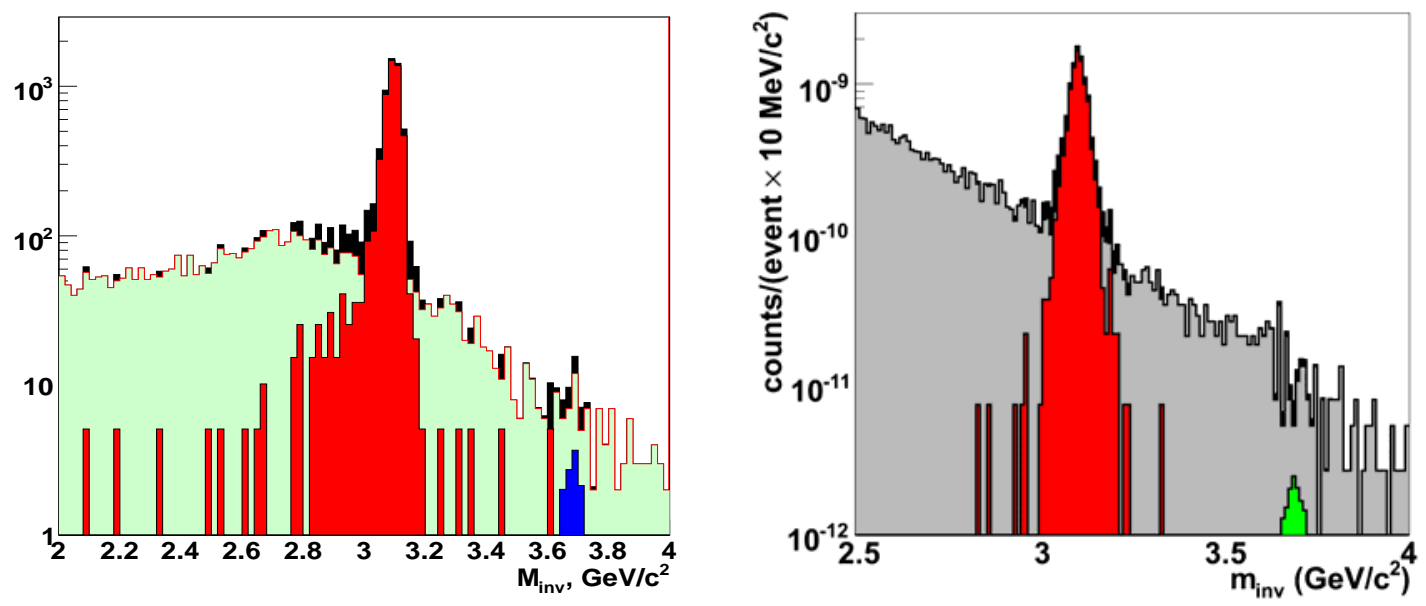

Figure 3. Invariant-mass spectra for pairs of identified electrons (left) and muons (right) in the charmonium mass region for central $\mathrm{Au}+\mathrm{Au}$ collisions at $25 \mathrm{~A} \mathrm{GeV}$

For the muon measurement, hadrons will be suppressed by a combination of absorbers and detector layers which allow tracking the particle trajectories from the STS through the system. Several detector options for this muon system are being investigated (GEM, straw tubes, MWPC). The background for the muon measurement mainly consists of muons from weak decays of pions and kaons, and of track mismatches. Figure 3 compares the anticipated performances of the charmonium measurement in the electron and the muon setup, which, despite of the different nature of the respective backgrounds, are quite similar.

\subsection{Low-mass vector mesons}

The properties of hadrons are expected to be modified in a dense hadronic environment by the restoration of chiral symmetry in the deonfined state. Their dileptonic decays of short-lived vector mesons $(\rho, \omega, \varphi)$ provide the tool to study such modifications since the lepton daughters will leave the dense medium undisturbed. CBM will measure both the $\mathrm{e}^{+} \mathrm{e}^{-}$and the $\mu^{+} \mu^{-}$decay channels, using the lepton identification devices as described above for the charmonium measurement. As in the case of charmonium, the simulated performances in both scenarios are comparable.

\section{Status of the experiment}

The FAIR project, first presented in a Conceptual Design Report in 2001, has meanwhile become an approved project. SIS-300 is expected to deliver beams from 2015 on.

From the beginning, the CBM experiment was considered a core experiment within the FAIR project, approved on the base of the Letter of Intent in January 2004. By today, the CBM collaboration consists of about 400 physicists from 46 institutions all over the world. The state of the preparations is laid down in the CBM Technical Status Report of 2005 [12] and, lately, the CBM Progress Report [13].

Performance studies leading to the final detector designs are ongoing together with detector R\&D, as briefly outlined in the previous sections, in order to reach the ambitious 
design specifications of CBM. The goal of these activities is to arrive at a Technical Proposal for the CBM experiment by $2011 / 2012$.

\section{References}

[1] F. Karsch, PoS(CPOD07)027

[2] http://www.gsi.de/fair/index_e.html

[3] http://Www.gsi.de/fair/experiments/CBM/indedx_e.html

[4] The CBM collaboration, CBM Technical Status Report, Darmstadt 2005, http://WwW.gsi.de/documents/DOC-2005-Feb-447-1.pdf

[5] The CBM Collaboration, CBM Progress Report 2006, Darmstadt 2007, http://www.gsi.de/documents/DOC-2007-Mar-137-1.pdf 